\title{
Spatial mapping of the Hanle and Zeeman effects on the Sun
}

\author{
J. O. Stenflo ${ }^{1}$, A. Gandorfer ${ }^{1}$, R. Holzreuter ${ }^{1}$, D. Gisler ${ }^{1}$, C. U. Keller ${ }^{2}$, and M. Bianda ${ }^{3}$ \\ 1 Institute of Astronomy, ETH Zentrum, 8092 Zurich, Switzerland \\ 2 National Solar Observatory, PO Box 26732, Tucson, AZ 85726-6732, USA \\ 3 Istituto Ricerche Solari Locarno (IRSOL), via Patocchi, 6605 Locarno-Monti, Switzerland
}

Received 27 February 2002 / Accepted 16 April 2002

\begin{abstract}
Spatial mapping of the Hanle and Zeeman effects on the Sun has been done for the first time, through Stokes vector imaging with a narrow-band $(0.2 \AA)$ universal filter. It is shown how the polarization signatures of the Hanle and Zeeman effects can be cleanly distinguished from each other by comparing the Stokes images recorded at different, specially selected wavelengths within the $\mathrm{Na}$ I $\mathrm{D}_{2}-\mathrm{D}_{1}$ line system. Examples of the polarization signatures of sunspots, faculae, the supergranulation network, and large-scale canopy fields are shown. The most striking result of our observations is that the scattering polarization has an extremely intermittent structure rather than being a simple function of limb distance. These intermittent scattering polarization signals are cospatial with the facular and supergranulation network seen both in intensity and circular polarization. The observed pattern can be explained in terms of magnetic enhancement of the scattering polarization in the network and/or Hanle depolarization of the scattering polarization outside the network. Since however no magnetic fields are seen in circular polarization outside the network, the relative absence of linear scattering polarization there may be explained by Hanle depolarization only if the volume filling, depolarizing magnetic field has mixed polarities on a subarcsec scale that is not resolved.
\end{abstract}

Key words. polarization - scattering - Sun: magnetic fields - atomic processes - techniques: polarimetric

\section{Introduction}

The linearly polarized solar spectrum that is produced by coherent scattering processes on the Sun has been called the "second solar spectrum" because of its structural richness with little semblance to the ordinary intensity spectrum. Because the polarized spectral structures were unfamiliar to astrophysics, the early work on the second solar spectrum has focused on exploratory investigations in the spectral domain, with emphasis on identification of the new spectral features and their underlying physical processes. The observational approach has therefore been to obtain 1-D Stokes $I$ and $Q / I$ spectra at discrete spatial locations near the solar limb with the highest possible polarimetric accuracy (about $10^{-5}$ and occasionally even better) in combination with high spectral resolution (Stenflo \& Keller 1997; Stenflo et al. 1997, 1998, 2000a,b; Gandorfer 2000; Trujillo Bueno et al. 2001; Arnaud et al. 2001; Bommier \& Molodij 2002).

At the same time it was clear that the information that is encoded in the second solar spectrum has great potential for magnetic-field diagnostics through the Hanle effect, which represents the influence of magnetic fields

Send offprint requests to: J. O. Stenflo,

e-mail: stenflo@astro.phys.ethg.ch on the scattering polarization. Since the magnetic fields are highly structured spatially and vary temporally, the polarization signatures should also exhibit such variations. Comparison of many recordings done at different spatial locations and at different times have shown that this is indeed the case, and that the fluctuations are actually larger than previously expected (Stenflo et al. 1998).

To explore the spatial structuring of the second solar spectrum we need to go from the 1-D to the 2-D case and also record the full Stokes vector, not just Stokes $I$ and $Q / I$. This became possible with the application of the second generation, ZIMPOL II, of the Zurich Imaging Polarimeter system (Stenflo et al. 1992; Povel 1995, 2001; Gandorfer \& Povel 1997; Gandorfer 1999). In the first application of ZIMPOLII, at the spectral focus of the McMath-Pierce facility of the National Solar Observatory (Kitt Peak), the polarization signatures of the Hanle and Zeeman effects could be clarified and distinguished from each other, through Stokes vector imaging covering the whole $\mathrm{Na}$ I $\mathrm{D}_{2}-\mathrm{D}_{1}$ line system simultaneously. In particular, it could be determined that the Hanle effect in the cores of the $D_{2}$ and $D_{1}$ lines are governed by different physics, for $\mathrm{D}_{2}$ primarily by upper-state atomic polarization, for $\mathrm{D}_{1}$ by ground-state atomic polarization induced by optical pumping in combination with hyperfine 
structure splitting (Stenflo et al. 2001). The concept of optical pumping as an explanation for the $\mathrm{D}_{1}$ polarization has been developed in ground-breaking theoretical work of Landi Degl'Innocenti $(1998,1999)$ and has since been worked out in greater detail by Trujillo Bueno et al. (2002).

After having provided clarifications of the spectral signatures of the Hanle and Zeeman effects in the $\mathrm{NaI} \mathrm{D}_{2}-$ $\mathrm{D}_{1}$ line system we are now in a position to address the interpretation of monochromatic Stokes images in these lines and to distinguish between the Hanle and Zeeman contributions. The earlier work has paved the way for explorations of the spatial morphologies of the Hanle and Zeeman effects and how they are interrelated.

In the present paper we apply ZIMPOL II for the first time with a narrow-band, universal filter system to record Stokes vector filtergrams of the Hanle and Zeeman effects at strategically selected wavelength positions within the $\mathrm{Na}$ I $\mathrm{D}_{2}-\mathrm{D}_{1}$ line system. This sodium multiplet is chosen for the following reasons: (i) It exhibits strong scattering polarization signals. (ii) The relatively large line widths better match the relatively broad filter pass band than is the case for most other scattering lines in the solar spectrum. (iii) This line system has a rich and intriguing spectral structure with considerable diagnostic potential. (iv) Its properties have been well explored in the spectral domain.

\section{Observations and data reduction}

The observations were carried out with ZIMPOL II on September 5 and 6, 2000, at the Dunn Solar Telescope of the National Solar Observatory (NSO) at Sacramento Peak. The Universal Birefringent Filter (UBF) with a pass band of $0.2 \AA$ was used for the wavelength selection.

With ZIMPOL II the four Stokes images are recorded by cycling the charges in the $\mathrm{CCD}$ sensor at $\mathrm{kHz}$ rates, in synchrony with the polarization modulation, between the exposed pixels and hidden fast buffers. Since the exposed pixels are the same for all four image planes, and since the cycling is done much faster than the seeing fluctuations, the fractional polarization images are entirely free from spurious seeing and gain-table effects.

To record all four Stokes images strictly simultaneously one would need two phase-locked polarization modulators. The present version of ZIMPOL II only uses one piezoelastic modulator, which means that only three Stokes images, $(I, V / I$, and either $Q / I$ or $U / I)$ are simultaneous. To obtain all four, we make sequential recordings, first of $I, Q / I$, and $V / I$, then the modulation package is rotated $45^{\circ}$ and $I, U / I$, and $V / I$ are recorded, after which the package is rotated back again.

The CCD was always oriented such that the pixel columns were parallel to the nearest solar limb (which was located just inside the field of view). The pixel size corresponded to 0.22 arcsec on the Sun. For each group of four pixel rows, however, three rows were masked to be used as hidden fast buffers to store the three image planes while the fourth was being exposed. Therefore the effective pixel size in the direction parallel to the limb was 0.88 arcsec. To obtain square resolution elements we do numerical binning in the row direction with a 4-pixel running window, which gives an effective pixel size of $0.88 \times 0.88 \operatorname{arcsec}^{2}$.

The filter pass band was set at a sequence of six discrete wavelength positions within the $\mathrm{Na}$ I $\mathrm{D}_{2}-\mathrm{D}_{1}$ line system. At each wavelength position the full Stokes vector (all four Stokes filtergrams, with alternating recordings of Stokes $Q$ and $U$, as indicated above) is recorded. After cycling through the six wavelengths, the procedure is repeated to enhance the polarimetric accuracy by averaging many measurements. With these repetitions, the time used for an observing run at a given spatial pointing has been $30 \mathrm{~min}$ for the observations presented here. This effective integration time can of course be chosen to be much smaller, to enhance time resolution at the expense of polarimetric accuracy. The choice is not limited by the instrumentation used (except for the short time the UBF needs to move from one wavelength position to the next, but this is done automatically, controlled by a computer script). In the present paper the trade-off is in favor of polarimetric accuracy.

The six selected wavelength positions are the following: The line centers of the $\mathrm{D}_{2}$ and $\mathrm{D}_{1}$ lines $\left(\lambda_{\mathrm{D} 2}\right.$ and $\left.\lambda_{\mathrm{D} 1}\right)$, the blue and red wings of the $\mathrm{D}_{2}$ line, at $\lambda_{\mathrm{bw}}=\lambda_{\mathrm{D} 2}-155 \mathrm{~m} \AA$ and $\lambda_{\mathrm{rw}}=\lambda_{\mathrm{D} 2}+155 \mathrm{~m} \AA$, the position in the blue wing where the non-magnetic scattering polarization in the $\mathrm{D}_{2}$ line has its maximum, at $\lambda_{\mathrm{D} 2}-0.41 \AA$, and a position in the continuum, at $\lambda_{\mathrm{D} 2}-7.77 \AA$ (5882.20 $\AA$ ). In Fig. 1 all these wavelength positions except for the continuum position are marked with thick, extended tick marks.

To keep our presentation focused we will here limit the discussion of the observational results to the four most relevant wavelengths, namely $\lambda_{\mathrm{D} 2}, \lambda_{\mathrm{D} 1}, \lambda_{\mathrm{bw}}$, and $\lambda_{\mathrm{rw}}$. The reason for the particular wavelength choices are the following: The Hanle effect is only present at the line center wavelengths and is greatly different between the $D_{2}$ and $\mathrm{D}_{1}$ lines, in contrast to the transverse Zeeman effect, for which the two lines behave the same. At $\pm 155 \mathrm{~m} \AA$ from line center the scattering polarization has deep minima (cf. Stenflo et al. 2000a), while the transverse Zeeman effect, taking into account the smoothing effect of the filter pass band, has its maximum there (cf. Fig. 1 in Stenflo et al. 2001). These wing positions are also nearly coinciding with the location of the maximum efficiency of the longitudinal Zeeman effect (as seen in $V / I$ ).

The rms noise level at line center (where the intensity level is an order of magnitude below the continuum level) for each pixel in the $Q / I$ and $V / I$ images is about $0.1 \%$. For the $U / I$ images the noise level was larger by a factor of 3.5 , which had to do with the polarizing properties of the UBF when combined with the polarized output from the modulation package. The throughput of the system was optimized for Stokes $Q$, which is of more relevance for the present work than Stokes $U$. When the modulator package was rotated $45^{\circ}$ for the Stokes $U$ recording, the polarizers of the modulator package and the UBF partially 


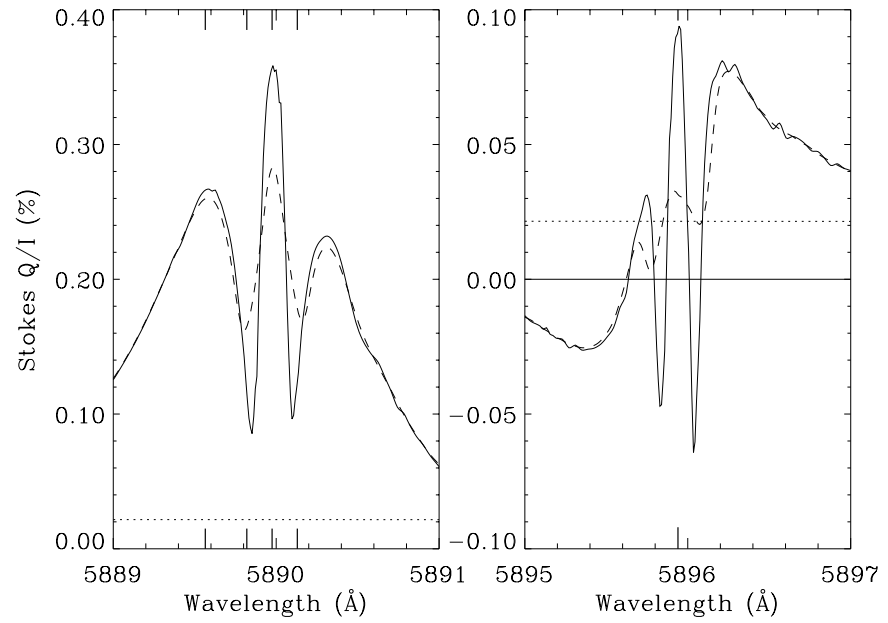

Fig. 1. The solid curves show the linearly polarized profiles (Stokes $Q / I$ ) recorded on the quiet Sun at $\mu=0.1$ by Stenflo et al. (2000a), for Na I $\mathrm{D}_{2}$ (left panel) and $\mathrm{D}_{1}$ (right panel). The dashed curves show the profiles after smearing with a boxcar window of width $0.2 \AA$, to illustrate the smearing effect of the filter used. The long and thick tick marks show five of the wavelengths at which the filter was positioned (the sixth position, for the continuum, falls outside the diagrams). The dotted line represents the level of the polarization for the adjacent continuum, according to the theory of Fluri \& Stenflo (1999). Note that the polarization scale of the $\mathrm{D}_{1}$ diagram has been expanded by a factor of two with respect to the scale of the $\mathrm{D}_{2}$ diagram.

cross, resulting in lower transmission and therefore higher noise level.

One major problem in vector polarimetry with the Dunn Solar Telescope (DST), like in our previous observations with the McMath-Pierce facility at NSO/Kitt Peak although substantially more severe in the case of the DST, is the large and rapidly varying instrumental polarization produced by several oblique reflections and in the entrance and exit windows of the vacuum system. Attempts at modelling the telescope polarization have shown that the cross-talk terms (non-diagonal elements of the instrumental Mueller matrix) cannot be determined with sufficient precision this way. Another method that we have tried is to do a Stokes vector recording in an active region with strong Zeeman-effect polarization signals just before and after the actual limb observations, and from regression analysis between the Stokes parameters in the active region determine the cross talk terms. This method is in most cases not accurate enough, in particular during times of the day when the instrumental polarization changes most rapidly. The problem of rapid variability may be avoided if regression analysis between the Stokes parameters can be made for the actual limb region recording. This method however works only if one has selected a limb region with considerable magnetic activity, so that there are strong polarization signals in all Stokes parameters.

For these reasons we find that the most reliable and accurate method to eliminate the instrumental cross talk is to make use of the qualitatively known properties of the scattering polarization and the Hanle and Zeeman effects in the data reduction, in particular their symmetry properties, as was done in Stenflo et al. (2001) for the Stokes vector recordings with the McMath-Pierce facility. This however works reliably only if one has a set of Stokes recordings at strategically selected wavelengths, which is the case in the present work. When planning an observing run for vector polarimetry it is essential to choose the observables such that such disentangling of the cross-talk terms does indeed become possible, since in general this is not the case. Fortunately, for well selected observables (like here), the polarization signatures of the Hanle and Zeeman effects are in a qualitative sense "orthogonal" to each other, which allows unambiguous cross-talk removal. In Sect. 3.1 below this unique distinction between the polarization signatures will be clarified. Let us here summarize the criteria used for the cross talk removal and refer to Sect. 3.1 and Fig. 2 for further clarifications and illustration of the principles.

Cross talk from Stokes $I$ to $Q, U, V$ is of no consequence here, since it only causes a spatially flat zero-line offset in $Q / I, U / I$, and $V / I$, without any spatial structuring. Of the remaining cross talks, the largest ones are $V \rightarrow Q$ and $V \rightarrow U$. They are eliminated by subtracting a fraction of the $V / I$ images from the $Q / I$ images, and another fraction from the $U / I$ images. These fractions are chosen so that no signatures of the longitudinal Zeeman effect appear in the $Q / I$ and $U / I$ images. These signatures are explained and illustrated in Sect. 3.1 and Fig. 2. $Q \rightarrow V$ cross talk is then eliminated by subtracting a fraction of the $Q / I$ images from $V / I$ such that the scattering polarization at the $\mathrm{D}_{2}$ line center and the transverse Zeeman effect pattern at both the $\mathrm{D}_{2}$ and $\mathrm{D}_{1}$ line centers do not appear in the corresponding $V / I$ images. Similarly, $Q \rightarrow U$ cross talk is eliminated by requiring that the scattering polarization pattern at the $\mathrm{D}_{2}$ line center is minimized in $U / I$ (part of the pattern could in principle appear with both signs in $U / I$ due to Hanle rotation, but with our criterion we minimize the single-sign scattering polarization pattern in $U / I)$. $U \rightarrow V$ cross talk is eliminated by minimizing the transverse Zeeman effect signals at the $\mathrm{D}_{2}$ and $\mathrm{D}_{1}$ line centers in the $V / I$ images.

For the removal of the remaining cross talk, $U \rightarrow Q$, we have no good criterion to use. This could be a problem for the determination of the correct azimuth angle of the magnetic fields from the transverse Zeeman effect. However, for the scattering polarization problem considered in the present paper it is of no consequence, since our images show no significant scattering polarization in $U / I$. Therefore we have refrained from applying any $U \rightarrow Q$ cross talk correction to our data.

\section{Results}

We will here present results for representative examples of limb regions with varying degrees of magnetic activity. Our first task is to bring out the distinctive 

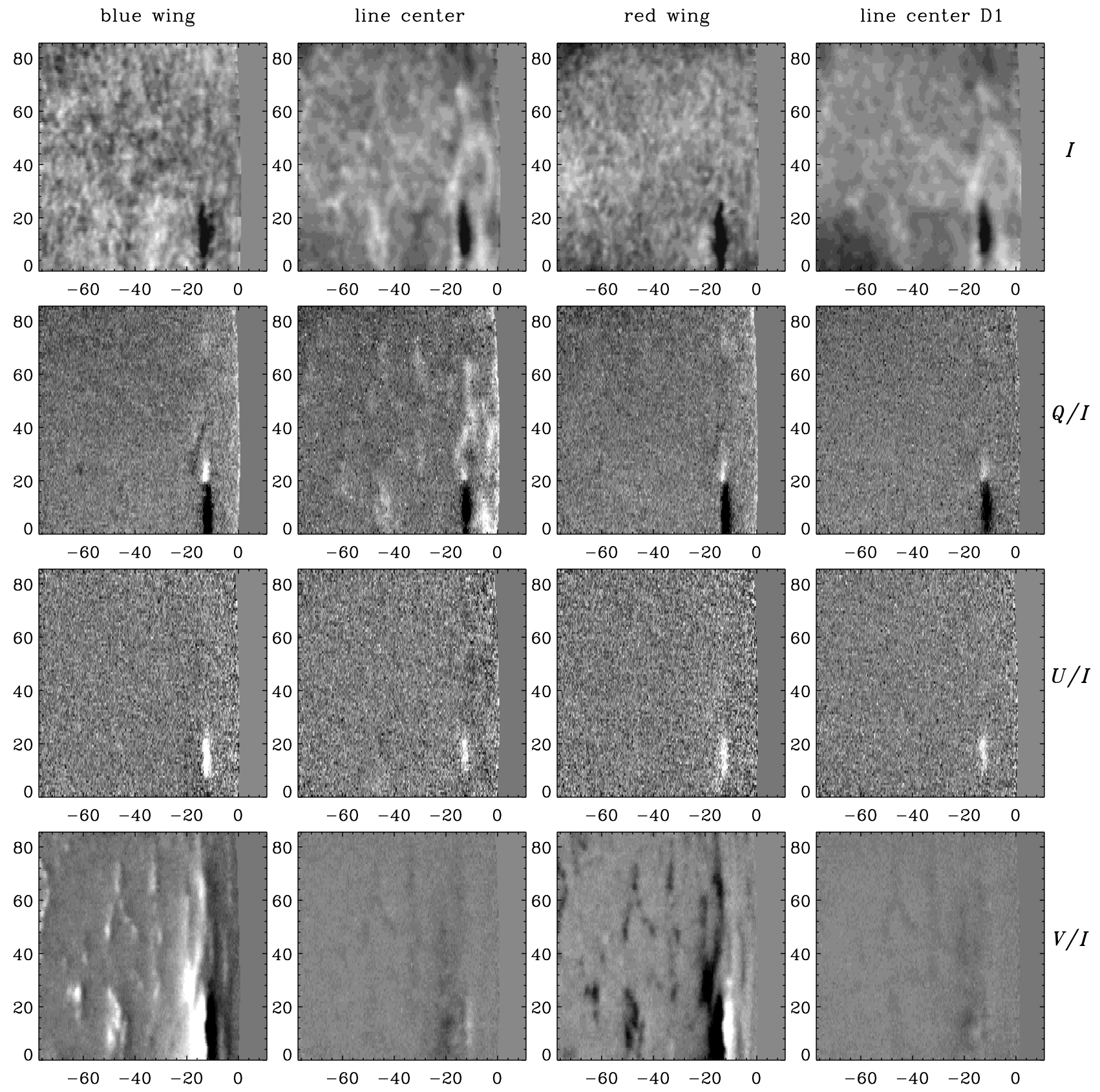

Fig. 2. Moderately active limb region with sunspot, recorded on September 6, 2000, at about $32^{\circ}$ heliographic north at the east limb. The units on the axes are arcsec. The zero point on the horizontal scale marks the position of the solar limb. The four rows represent the four Stokes parameters $I, Q / I, U / I$, and $V / I$. The first three columns refer to the three wavelengths in the $D_{2}$ line, the fourth column to the line center of $D_{1}$. The blue and red wing positions are at $\mp 155 \mathrm{~m} \AA$ from the $D_{2}$ line center. The cuts for the grey scale are the same for the four images within a given row. The Stokes $I$ images have been normalized to the average limb darkening function for the respective wavelength, to bring out the local intensity structures with good contrast. The grey scale minimum and maximum (black and white) for the Stokes $I$ image are $1.0 \pm 0.2$, in terms of the mentioned normalization. For the polarization images the grey scale limits are in terms of degree polarization: For $Q / I \pm 0.6 \%$, for $U / I \pm 1.4 \%$, for $V / I \pm 2.0 \%$. The choice of larger limits for $U$ as compared with $Q$ has to do with the 3.5 times larger noise in the $U / I$ images (cf. Sect. 2), which would be enhanced too much with smaller cuts.

characteristics of the Hanle and Zeeman effects to understand what the Stokes images are really telling us. Having identified the physical mechanisms, the next step is to interpret the structures we see and their implications for our understanding of the nature of solar magnetic fields. As we will see, these Stokes images may constrain the properties of solar magnetic fields in ways not possible with the Zeeman effect alone, for instance the spatially unresolved properties of the non-network fields.

Figure 2 shows a region at the east limb, which was inconspicuous in $\mathrm{H} \alpha$, but in the sodium images reveals a geometrically foreshortened sunspot at about $13 \operatorname{arcsec}$ 
from the limb (where the cosine of the heliocentric angle, $\mu \approx 0.16$ ). The region exhibits facular activity, which in the intensity images (Stokes $I$ ) however only shows up well in the line core images. The emission pattern in the line core, which is formed in the lower chromosphere, has a softer and coarser appearance as compared with the more granular, smaller-scale pattern in the deeper-forming line wings. The facular emission in the line cores has a networklike structure.

The Stokes $V / I$ signals are exclusively due to the longitudinal Zeeman effect, since scattering polarization and the Hanle effect only appear in the linear polarization. As expected, the $V / I$ signal shows up in the line wings and is almost zero at line center. Small residual signals at line center can occur if the line is not centered on the filter pass band (e.g. due to varying Doppler shifts on the Sun), or if the filter and/or Stokes $V / I$ profiles are not symmetric/anti-symmetric. Another characteristic feature of the longitudinal Zeeman effect is that the signals in the blue and red line wings have opposite signs, which is clearly seen in all our recordings.

\subsection{Distinction between the Hanle and Zeeman structures}

Since Stokes $V / I$ is the exclusive domain of the longitudinal Zeeman effect, no confusion between the Hanle and Zeeman effect is possible there. The situation is however different for Stokes $Q / I$ and $U / I$, since both the transverse Zeeman effect and the Hanle effect can be the source of variable linear polarization. The issue in this section is therefore how we can know if a structure seen in the $Q / I$ or $U / I$ image is due to the Zeeman and/or the Hanle effect.

Such a distinction is not possible if only one wavelength position is used. However, for certain choices of combinations of wavelengths, an unambiguous separation is possible. We will use Fig. 2 to illustrate this. The same discussion also applies to the other figures.

As we have seen from our exploration of the spectral domain of the $\mathrm{Na}$ I $\mathrm{D}_{2}-\mathrm{D}_{1}$ line system (Stenflo et al. 2001) the polarization signals from the pure Zeeman effect (when the Hanle effect can safely be neglected, like in a sunspot region far from the limb) are of very similar amplitudes in the $D_{2}$ and $D_{1}$ lines (cf. Fig. 1 of Stenflo et al. 2001). In contrast, the scattering polarization is much larger in $\mathrm{D}_{2}$ than in $\mathrm{D}_{1}$. With infinite spectral resolution the $\mathrm{D}_{2}$ $Q / I$ core peak would be $3-4$ times larger than the $\mathrm{D}_{1}$ core peak (Stenflo et al. 2000a). Since our filter pass band is about twice as wide as these core peaks, and as the $\mathrm{D}_{1}$ core peak is somewhat narrower than the $\mathrm{D}_{2}$ peak and in addition is surrounded by sharp, negative minima, the scattering polarization signal in the $\mathrm{D}_{1}$ core is expected to be practically vanishing with a filter pass band as wide as ours. To resolve the $\mathrm{D}_{1}$ structure, a substantially narrower filter (by a factor of two or more) would be needed.

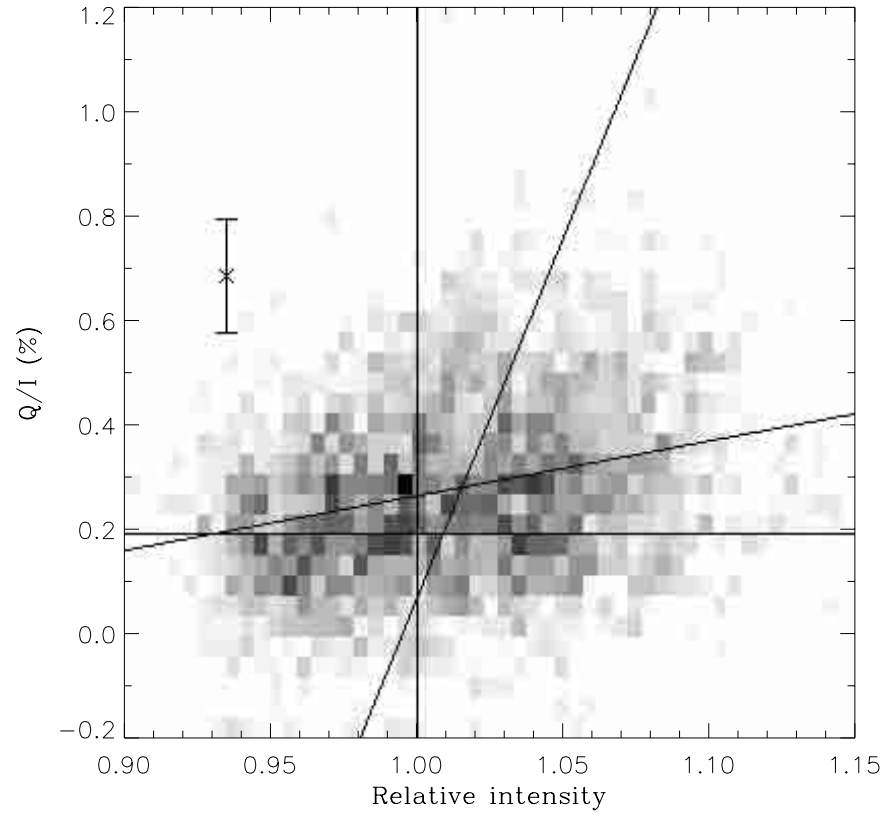

Fig. 3. Illustration of the degree of correlation between polarization $(Q / I)$ and intensity for a selected region in a narrow limb-distance interval above the sunspot in Fig. 2. The grey scale gives the number density of points in the scatter plot. The dashed lines are the two regression lines ( $y$ vs. $x$ and $x$ vs. $y$ ). The vertical line indicates the mean intensity at this limb distance, the horizontal, fiducial line the characteristic $Q / I$ level outside the network. The error bar $( \pm 0.1 \%)$ is also given, to the upper left. If the points were uncorrelated, the two regression lines would be $90^{\circ}$ apart.

According to the sunspot Stokes spectra in Fig. 1 of Stenflo et al. (2001) the $Q / I$ and $U / I$ maxima of the dominating $\sigma$ components are about $0.13 \AA$ from line center, while the $\pi$ component at line center is almost invisible. With the $0.2 \AA$ pass band we therefore expect to partially pick up the $\sigma$ component signals also when the pass band is positioned at line center, and will therefore see the same sign of the transverse Zeeman signal at all our selected wavelength positions. This is indeed the case, as shown here by Figs. 2 and 4, 5.

When inspecting the $Q / I$ and $U / I$ images in Fig. 2 we can distinguish between two patterns. One pattern is associated only with the sunspot and is seen with similar strength at all four wavelength positions. In the $Q / I$ images it appears with both signs (black and white), in $U / I$ only with positive (white) sign. This pattern, for instance as revealed in the $\mathrm{D}_{1}$ core images, is therefore exclusively due to the transverse Zeeman effect. The second pattern is seen only in the $Q / I \mathrm{D}_{2}$ line center image, but it is absent in all the other 7 linear polarization images of Fig. 2. It is therefore exclusively due to scattering polarization with the Hanle effect. This $\mathrm{D}_{2} Q / I$ pattern is cospatial with the emission network seen in the $\mathrm{D}_{2}$ and $\mathrm{D}_{1}$ Stokes $I$ images.

To get a feeling for the degree of correlation between the line center $Q / I$ and $I$ patterns we plot in Fig. $3 Q / I$ vs. $I$ for a selected rectangular area in Fig. 2 just above the sunpot but at the same limb distance as the spot, 

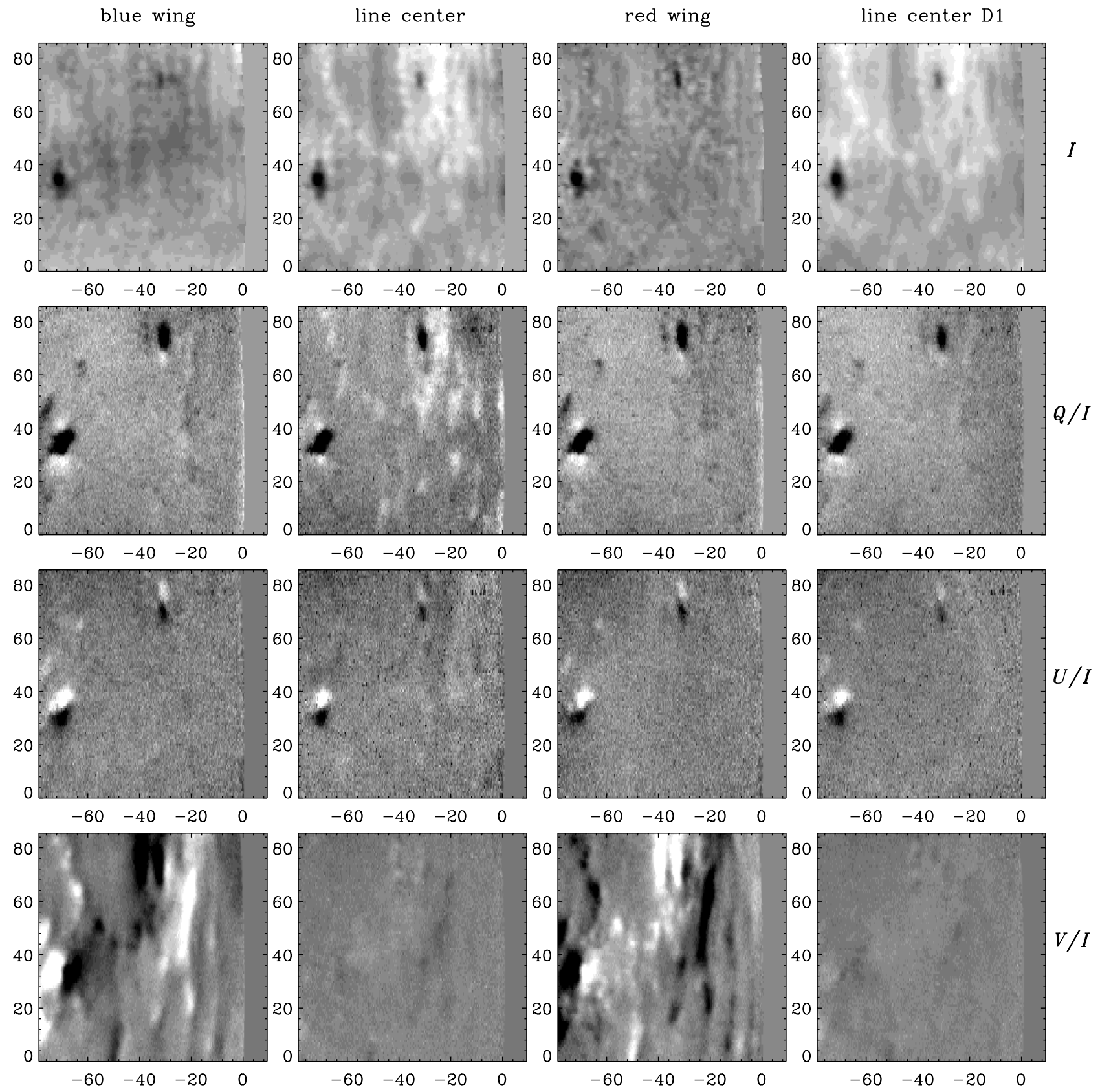

Fig. 4. Stokes vector recording like in Fig. 2, also obtained at the east limb on September 6, 2000, but at $22^{\circ}$ heliographic north. The grey scale cuts were: For $Q / I \pm 0.7 \%$, for $U / I \pm 1.4 \%$, for $V / I \pm 2.5 \%$.

where polarizing network structures are seen. For such a plot the selected solar region has to be narrow, to avoid the confusing effect of the very steep center-to-limb variation in $Q / I$. For perfect correlation, the two regression lines would coincide, while for zero correlation they would be $90^{\circ}$ apart (horizontal and vertical, respectively). The two regression lines show that there is a clear, although fairly modest correlation. A substantial part of the scatter degrading the correlation is however due to the instrumental noise $( \pm 0.1 \%$ in $Q / I$, as indicated by the error bar in Fig. 3).

The same type of discussion also applies to Fig. 4, which like Fig. 2 was obtained at the east limb, but $10^{\circ}$ closer to the equator (at about $22^{\circ}$ heliographic north), where the level of magnetic activity was somewhat higher. This region has stronger facular activity and two sunspots with prominent signals of the transverse Zeeman effect. Note that the scattering polarization in $Q / I$ in the $\mathrm{D}_{2}$ line core shows a pattern that closely correlates with the facular emission network seen in Stokes $I$.

\subsection{Magnetic-field diagnostics with the Hanle effect}

The transverse Zeeman-effect signals are only significant at the location of the sunspot, but are too weak to be seen in the rest of the field of view. In contrast, the longitudinal 

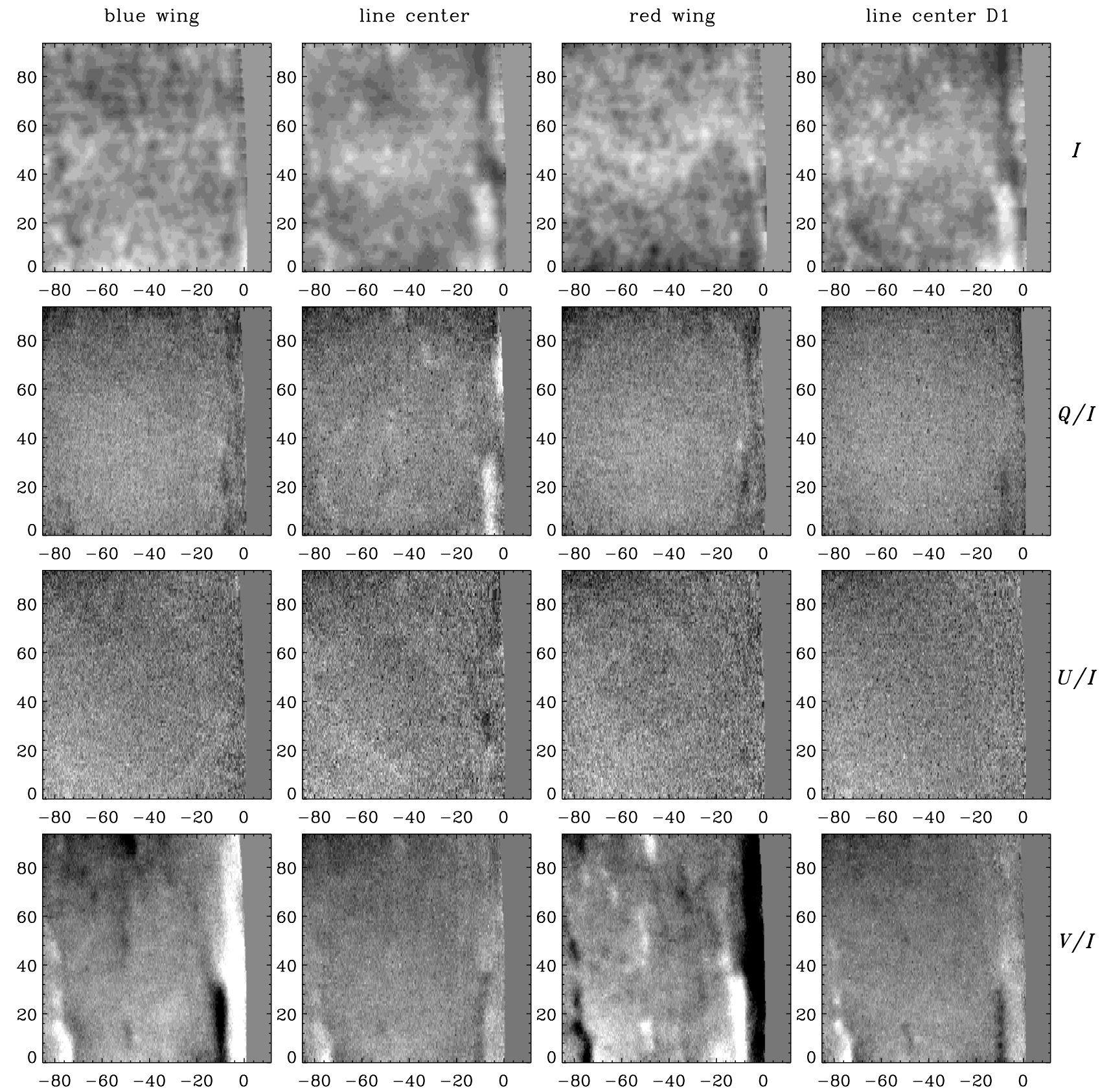

Fig. 5. Stokes recording like in Figs. 2 and 4, but obtained on September 5, 2000, at the west solar limb at about $22^{\circ}$ heliographic south in a semi-quiet region with some minor facular activity. Note the very pronounced canopy signature in $V / I$ in a 5 arcsec wide band that extends throughout the whole 1.5 arcmin field of view without sign reversal. The grey scale cuts were: For $Q / I \pm 0.5 \%$, for $U / I \pm 1.5 \%$, for $V / I \pm 0.8 \%$.

Zeeman effect in $V / I$ as well as the scattering polarization in the $\mathrm{D}_{2}$ line core exhibit signals all over the field of view. Since the amplitude of the scattering polarization decreases steeply with limb distance due to the rapidly changing scattering geometry, the pattern becomes quite weak in the left portion of the field of view (at about one arcmin from the limb), although it is still discernible there.

The $V / I$ signal is a measure of the line-of-sight component of the magnetic field, but it does not tell about the orientation of the field vector. The scattering polarization on the other hand depends in a complex way on both the strength and direction of the field, but since it has two observables $(Q / I$ and $U / I)$, while the field has three vector components, the field vector cannot be uniquely constrained by the scattering polarization alone. Still the partial constraints allow important conclusions about the field structure, as we will see.

For the scattering geometry near the solar limb, which qualitatively resembles the case of $90^{\circ}$ scattering, magnetic fields suppress the scattering polarization, but they should not enhance it according to standard Hanle effect theory (in contrast to the case closer to disk 
center, when the scattering geometry qualitatively resembles the forward-scattering case, cf. Trujillo Bueno 2001). The largest polarization amplitudes near the limb would then be expected for the non-magnetic case. It may therefore appear paradoxical and contradictory that the scattering polarization pattern that we see in $Q / I$ for the $\mathrm{D}_{2}$ line center is correlated with the Stokes $I$ emission network (cf. Fig. 3), where we expect the largest magnetic-field concentrations. The $V / I$ pattern is also found to be correlated with the emission network. Inspection of our Stokes vector images indicates that the scattering polarization is strongest where also the magnetic flux concentration is the strongest.

Part of the resolution of this paradox has to do with the angular dependence of the Hanle effect. The Hanle depolarization is largest for horizontal fields, but it vanishes for vertical fields, regardless of the orientation of the line of sight, because the illumination of the scattering particles is symmetric around the field vector when the field is vertical. If the field orientation in the network is close to vertical, as expected because of the strong buoyancy forces when the flux tubes have $\mathrm{kG}$ strengths, then we should also expect to find the largest scattering polarization in the network, as our observations indeed show. An additional effect is the recent finding by Trujillo Bueno et al. (2002) that for a vertical magnetic field the scattering polarization in the $\mathrm{D}_{2}$ line will actually increase with field strength for $B \gtrsim 10 \mathrm{G}$, as the Zeeman splitting becomes comparable to or larger than the hyperfine structure splitting and the damping widths of the excited sublevels. This is another reason why the vertical field regions will stand out in terms of scattering polarization.

Still the high contrast of the $Q / I$ network combined with the relative absence of $Q / I$ scattering polarization outside it suggests that this pattern may not be explainable only in terms of network enhancement, but that in addition a depolarizing mechanism is at work outside the network, although these regions look empty and field free in the $V / I$ images. If they really were field free, then there would be no Hanle suppression of the scattering polarization amplitudes, and we would expect to find $Q / I$ amplitudes outside the network more comparable in magnitude to the amplitudes within the network. Since the Hanle sensitivity range for the $\mathrm{D}_{2}$ line is centered around a field strength of about $5 \mathrm{G}$, Hanle depolarization in the nonnetwork can only happen if a considerable fraction of the volume is filled with fields of high inclination and with a field strength of about $5 \mathrm{G}$ or more. Such fields would however be visible also in our $V / I$ images, unless they have mixed orientations on a spatial scale that is smaller than our spatial resolution. We thus see how the combination of Stokes images of the Hanle and Zeeman effects can place qualitatively new types of constraints on the field, not possible with Zeeman diagnostics alone. Quantitative conclusions however require a radiative-transfer analysis that accounts for the above-mentioned magnetic-field enhancing effects, and this is outside the scope of the present paper.
With filter pass bands of $0.1 \AA$ or smaller it should be possible to see scattering polarization signals in the $D_{1}$ line as well. Since the $\mathrm{D}_{1}$ polarization is governed by lowerlevel atomic polarization and therefore responds to magnetic fields in a field-strength range that is about 100 times weaker as compared with the Hanle-effect range for the $\mathrm{D}_{2}$ line, the combination of the observed $\mathrm{D}_{2}$ and $\mathrm{D}_{1}$ scattering polarization patterns would bring an additional dimension to the diagnostic possibilities. It is therefore important to develop future observing systems with such a capability.

\subsection{Canopy signatures in a semi-quiet region}

One of the limb recordings (Fig. 5), on the west limb at about $22^{\circ}$ heliographic south in a region devoid of sunspots and with only very minor facular activity, showed an unexpected and very strong and large-scale canopy signature in the longitudinal Zeeman effect. Inspection of Kitt Peak magnetograms shows that this feature is related to the remnants of an old active region that just disappeared behind the west limb. As shown by Fig. 5, the strong and unipolar $V / I$ signal in a narrow ( 5 arcsec wide) band along the solar limb throughout the whole field of view (1.5 arcmin) is direct evidence for horizontal magnetic fields that remain coherent over a spatial scale of at least three supergranules. The reality of this limb zone band as being caused by the longitudinal Zeeman effect cannot be in doubt, since the $V / I$ patterns have opposite signs in the blue and red line wings, which is the characteristic anti-symmetric signature of the longitudinal Zeeman effect. Since this limb zone is confined to $\mu \lesssim 0.12$, the lineof-sight component of the field (as represented by $V / I$ ) is very close to horizontal. The $V / I$ polarization amplitudes of $\pm 0.8 \%$ correspond to an estimated field strength (as derived from the effective Landé factor and the intensity gradient $\partial I / \partial \lambda$ convolved with the filter pass band) of $25-30 \mathrm{G}$, which is somewhat stronger, but still not very different from the average canopy field strengths of $5-15 \mathrm{G}$ derived from the Hanle effect by Bianda et al. (1998a,b, 1999).

In the lower portion of the limb region there is an abrupt polarity reversal between the limb band and an opposite-polarity band immediately to the left of it. In both of these bands the field has strong horizontal components, but with opposite orientations. Exactly at the location between these two bands, where the polarity reverses, there is a strong scattering-polarization signal in the $\mathrm{D}_{2} Q / I$ line center image. This is evidence for at least partial absence of Hanle depolarization and possibly also magnetic enhancement of the scattering polarization, conditions that are best fulfilled if the field is vertically oriented there. A vertical field would imply that the polarity reversal does not occur through rotation of the field vector in the horizontal plane, but rather through rotation in a vertical plane, since this would allow the field to spend part of the time, between $4-8$ arcsec from the limb, in a nearly vertical form to give rise to such a large-contrast 
signal in the $Q / I$ image. If the reversal would instead occur through rotation in the horizontal plane, we would also expect to see an enhancement of the scattering polarization when the field vector is nearly perpendicular to the line of sight, because then the Hanle effect is reduced by $50 \%$. However, the expected signal would then be expected to be at most half as large as in the case of a vertical field. The high contrast of the observed $Q / I$ feature speaks in favor of a vertical field, but a definite conclusion would require a more quantitative analysis, which is outside the scope of the present paper.

\subsection{Hanle-Zeeman pattern in a quiet limb region}

In Fig. 6 we show a recording in the most quiet limb region that we could find that day (September 6, 2000), which was located on the west limb at $68^{\circ}$ heliographic north. Only the $Q / I$ and $V / I$ images are shown here, since the Stokes $I$ and $U / I$ images were structureless due to the very low level of magnetic activity. Still $V / I$ shows a remarkable number density of small-scale flux elements of mixed polarities, all the way to the limb. For $Q / I$ we can distinguish between two components: (1) A component that only varies with limb distance. It manifests itself as an increase in $Q / I$ near the limb. The increase is very slow far from the limb but increases in steepness as we approach the limb and ends with a bright and narrow band along the limb. It is seen in both the core and wings of the $\mathrm{D}_{2}$ line but is very weak and nearly absent in the $\mathrm{D}_{1}$ line. We note that this component was much less visible in the magnetically active regions (Figs. 2 and 4, 5). (2) A component consisting of a pattern of bright spots of positive scattering polarization, which is only seen in the $\mathrm{D}_{2}$ line center image. The locations of these bright spots are well correlated with the magnetic flux elements seen in the $V / I$ images. According to our previous arguments (cf. Sect. 3.2), this would indicate that the bright spots are locations of vertically oriented magnetic fields. While the Hanle depolarization vanishes for vertical magnetic fields, such fields may also enhance the scattering polarization in the $\mathrm{D}_{2}$ line beyond the non-magnetic scattering case, as shown by Trujillo Bueno et al. (2002). However, the relative absence of scattering polarization in the non-network background may also imply that the polarization there is suppressed by a highly inclined magnetic field that must have a strength of about $5 \mathrm{G}$ or more for the Hanle effect to be effective. Since this field is invisible in the $V / I \mathrm{im}-$ ages, while the visible flux patches that we see in Fig. 6 have typical field strengths of about $10 \mathrm{G}$ (as averaged over the spatial resolution element), we certainly have sufficient polarimetric sensitivity to see this non-network field if it were spatially resolved. If there is indeed Hanle reduction of the $Q / I$ signals in regions with no $V / I$ signal, then the field that is responsible for this Hanle depolarization must have mixed polarities on a subarcsec scale, in order to make the net $V / I$ polarization when averaged over the spatial resolution element nearly vanish.
We note that since none of the $Q / I$ structures seen in the $\mathrm{D}_{2}$ core is present in the $\mathrm{D}_{1}$ core, none of them can be caused by the transverse Zeeman effect, but all, without exception, are due to scattering polarization.

\section{Conclusions}

We have seen that with our choice of wavelength positions for the filter pass band and the use of two lines of the same multiplet it is possible to unambiguously distinguish between the polarization signals from the Zeeman and Hanle effects. These characteristic signatures are exploited to remove all the cross talk between the Stokes parameters due to instrumental polarization. We find that the scattering polarization in the line core has two components: One component that is a steep function of limb distance, and one component that is spatially structured and strongly correlated with the emission network and with the pattern of line-of-sight magnetic fluxes seen in $V / I$ (the longitudinal magnetograms). Unexpectedly, this latter component is the one that is the most conspicuous.

The most striking property of the Hanle-effect pattern is thus that most of the solar surface forms a lowpolarization background, on top of which islands of polarization that are cospatial with the supergranulation network appear. These islands have a rather small filling factor. When as in the past one derives 1-D polarization spectra by spatial averaging along the slit like in Stenflo et al. (2000a), we get for the line core an average between the polarizing network and the low-polarization background, weighted by their respective filling factors. The more distant line wings, like the $\mathrm{D}_{2}$ blue wing $Q / I$ maximum at $-0.41 \AA$ from the line center, are however not affected, since the magnetic field effects and the spatial structuring vanish in the wings.

If we had been dealing with a simpler atomic transition without hyperfine structure splitting and optical pumping, like the Sr I $4607 \AA$ scattering transition, the interpretation of the scattering polarization pattern would have been rather straightforward, since for scattering near the Sun's limb magnetic fields would only reduce the amount of scattering polarization relative to the non-magnetic case. In the present case the interpretation is however substantially more complex, because for $\mathrm{Na}_{\mathrm{I}} \mathrm{D}_{2}$ magnetic fields can both enhance and destroy the line core scattering polarization. The enhancement is caused by interferences between the sublevels of the excited state as the Zeeman splitting becomes comparable to or larger than the hyperfine structure splitting and the natural line widths (Trujillo Bueno et al. 2002). The largest polarization values are expected when the field is vertical. The destruction of the polarization is caused by the usual Hanle depolarization when inclined fields are present.

If there is to be a significant contribution of Hanle depolarization to account for the low-polarization nonnetwork background, then the field strength of the volume-filling, inclined fields should be at least about $5 \mathrm{G}$. Such fields would however easily be seen in the 

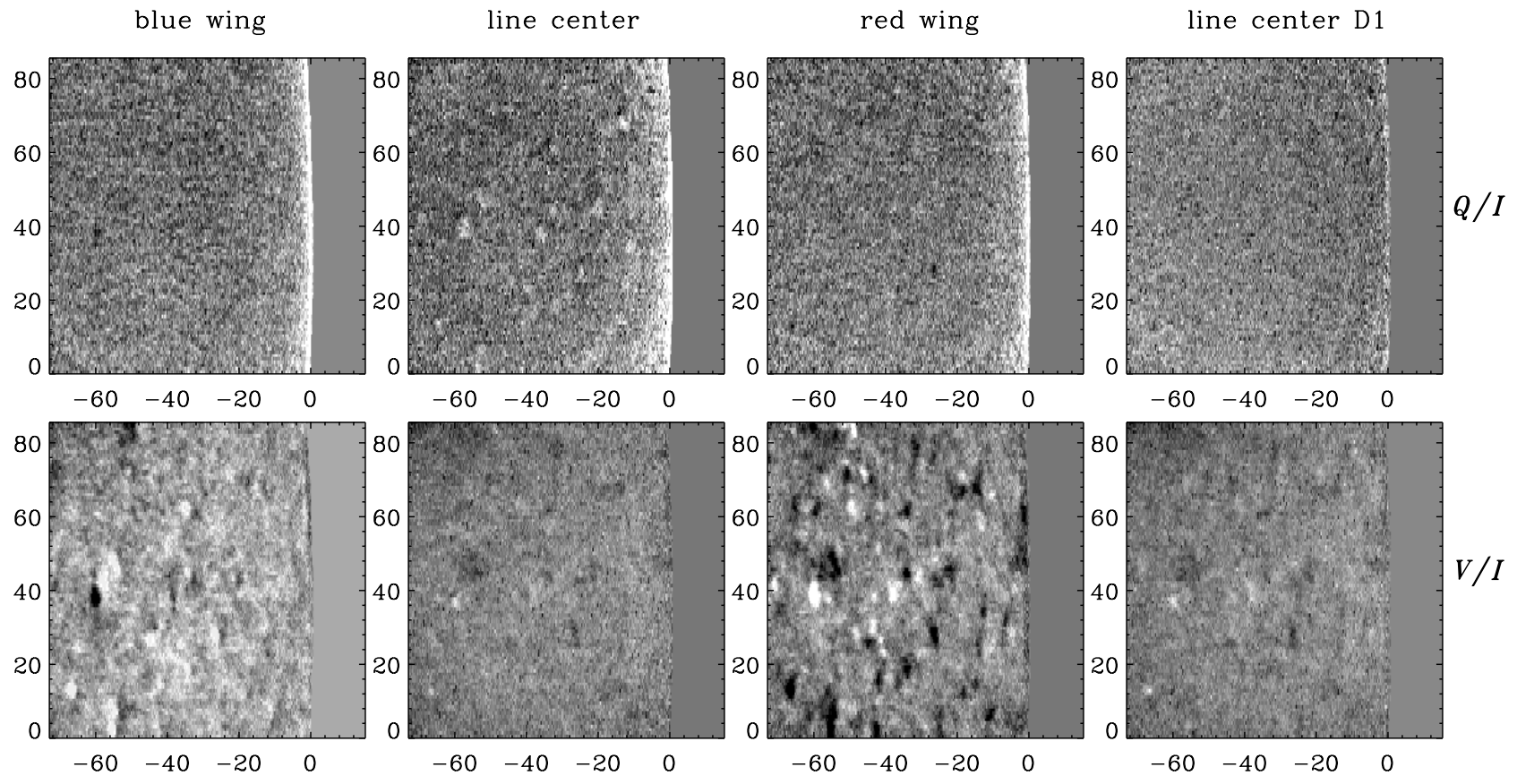

Fig. 6. Stokes images for a very quiet limb region, located at the geographical north, i.e., $22^{\circ}$ west of heliographic north. The recordings were made on September 6, 2000. Since this region was so quiet that the Stokes $I$ and $U / I$ images were featureless, we here only show the $Q / I$ and $V / I$ images. The grey scale cuts were: For $Q / I \pm 0.25 \%$, for $V / I \pm 0.4 \%$.

circular-polarization images if the fields were spatially resolved. Since they are not seen, Hanle depolarization can only occur if the fields are hidden from view, as happens when the magnetic polarities are mixed on a scale that is not resolved, and the $V / I$ signals cancel within the spatialresolution element.

We cannot here allocate the relative importance of magnetic enhancement in the network and Hanle depolarization outside the network without a quantitative radiative transfer treatment, which is far beyond the scope of the present paper, in particular since the novel effects of coherences within the $\mathrm{NaI} \mathrm{D}_{2}-\mathrm{D}_{1}$ system have not yet been incorporated in a radiative-transfer formalism. Since most spectral lines with scattering polarization would not be subject to the magnetic enhancement, which is rather special for the $\mathrm{D}_{2}$ line, observations in other spectral lines could clarify the role of Hanle depolarization outside the network. A practical obstacle here is that most such lines are substantially narrower than the $\mathrm{D}_{2}$ line and would therefore be too "washed out" by the broad $(0.2 \AA)$ filter pass band. Although earlier observations of scattering polarization have pointed to the presence of a ubiquitous Hanle depolarization background on the Sun, filter observations would be highly desirable to clarify its nature. This however requires the application of filters with substantially narrower pass bands.

The existence of a volume-filling, spatially unresolved mixed-polarity magnetic field with no visible Zeemaneffect signatures was in fact postulated already 20 years ago (Stenflo 1982) to explain the scattering-polarization observations that were available at that time (Stenflo et al. 1980). The strength of this field was constrained to be
10-100 G (Stenflo 1982). Later detailed radiative-transfer modelling by Faurobert-Scholl (1993), Faurobert-Scholl et al. (1995), and Faurobert et al. (2001) of observations in the Sr I $4607 \AA$ line allowed the field-strength range to be narrowed down to $10-30 \mathrm{G}$. The observations however also indicate that the turbulent field varies both spatially and temporally, in particular with the phase of the solar cycle (Stenflo et al. 1998; Faurobert et al. 2001). Note that the field-strength values given are based on an interpretation with a single-valued field. In reality we expect to have a continuous distribution of field strengths (cf. Stenflo 1999).

Although much progress has been made in the theoretical understanding of the scattering polarization of the $\mathrm{NaI} \mathrm{D}_{2}-\mathrm{D}_{1}$ line system, in particular through the work of Landi Degl'Innocenti $(1998,1999)$ and Trujillo Bueno et al. (2002), none of the theories presented so far has been able to reproduce the most frequently observed shape of the $Q / I$ profiles of the $\mathrm{D}_{1}$ line as given in Stenflo et al. (2000a). These profiles show a pronounced and narrow core peak surrounded by very sharp local minima. It is very possible that partial frequency redistribution plays an essential role in determining this line shape, but this has yet to be demonstrated. Before we can make better fits of such qualitative features for the $Q / I$ profiles of the $\mathrm{D}_{1}$ line, we cannot have full confidence that all the physics underlying the formation of the $\mathrm{NaI} \mathrm{D}_{2}-\mathrm{D}_{1}$ line system is yet fully understood. Further filter observations would help clarify the mechanisms involved and thereby guide the theory.

The pass band of the filter that was used was unfortunately too wide to resolve the narrow scattering 
polarization features in the $\mathrm{D}_{1}$ line. Since lower-level atomic polarization generated by optical pumping is needed to produce these features, the $\mathrm{D}_{1}$ polarization responds to magnetic fields already in the milligauss range, three orders of magnitude below the sensitivity range of the $\mathrm{D}_{2}$ line, as indicated empirically in Stenflo et al. (2001) and explored theoretically by Trujillo Bueno et al. (2002). If the $\mathrm{D}_{1}$ core polarization could be spectrally resolved, it would add a new diagnostic dimension to the exploration of the non-network fields as well as help us to sort out the different physical mechanisms. For this to be possible, high-precision vector polarimetry with tunable filters with a pass band of $0.1 \AA$ or less is needed. Such filters would open the door to diagnostic exploitation of the numerous other scattering lines in the solar spectrum. By combining lines that are governed by different atomic physics and formed in different ways in the solar atmosphere, we may resolve the present ambiguities in the interpretation and better use the rich diagnostic potential of the diverse magnetic-field signatures in the second solar spectrum.

Acknowledgements. The observations were carried out with ZIMPOL at the National Solar Observatory/Sacramento Peak. The engineering group at ETH Zurich (Peter Povel, Peter Steiner, Urs Egger, Frieder Aebersold, Stefan Hagenbuch) built the ZIMPOL system and provided the technical support. The ZIMPOL development program and two of the authors (A.G. and D.G.) have been funded by the Swiss Nationalfonds, grant No. 20-56853.99. NSO is one of the National Optical Astronomy Observatories, which are operated by the Association of Universities for Research in Astronomy, Inc. (AURA) under cooperative agreement with the National Science Foundation. We also thank Veronique Bommier for helpful comments on the manuscript.

\section{References}

Arnaud, J., Vigneau, J., Faurobert, M., \& Paletou, F. 2001, in Advanced Solar Polarimetry: Theory, Observations and Instrumentation, ed. M. Sigwarth, ASP Conf. Ser., 236, 151

Bianda, M., Solanki, S. K., \& Stenflo, J. O. 1998a, A\&A, 331, 760

Bianda, M., Stenflo, J. O., \& Solanki, S. K. 1998b, A\&A, 337, 565

Bianda, M., Stenflo, J. O., \& Solanki, S. K. 1999, A\&A, 350, 1060
Bommier, V., \& Molodij, G. 2002, A\&A, 381, 241

Faurobert-Scholl, M. 1993, A\&A, 268, 765

Faurobert-Scholl, M., Feautrier, N., Machefert, F., Petrovay, K., \& Spielfiedel, A. 1995, A\&A, 298, 289

Faurobert, M., Arnaud, J., Vigneau, J., \& Frisch, H. 2001, A\&A, 378, 627

Fluri, D. M., \& Stenflo, J. O. 1999, A\&A, 341, 902

Gandorfer, A. 1999, Opt. Eng., 38, 1402

Gandorfer, A. 2000, The Second Solar Spectrum, vol. I: $4625 \AA$ to $6995 \AA$, ISBN No. 3728127647 (Zurich: VdF)

Gandorfer, A., \& Povel, H. 1997, A\&A, 328, 381

Landi Degl'Innocenti, E. 1998, Nature, 392, 256

Landi Degl'Innocenti, E. 1999, in Proc. 2nd SPW, Solar Polarization, ed. K. N. Nagendra, \& J. O. Stenflo, ASSL (Dordrecht: Kluwer), 243, 61

Povel, H. P. 1995, Opt. Eng. 34, 1870

Povel, H. P. 2001, in Magnetic Fields across the HertzsprungRussel Diagram, ed. G. Mathys, S. K. Solanki, \& D. T. Wickramasinghe, ASP Conf. Ser., 248, 543

Stenflo, J. O. 1982, Solar Phys., 80, 209

Stenflo, J. O. 1999, in Proc. 2nd SPW, Solar Polarization, ed. K. N. Nagendra, \& J. O. Stenflo, ASSL (Dordrecht: Kluwer), 243, 1

Stenflo, J. O., \& Keller, C. U. 1997, A\&A, 321, 927

Stenflo, J. O., Baur, T. G., \& Elmore, D. F. 1980, A\&A, 84,60

Stenflo, J. O., Keller, C. U., \& Povel, H. P. 1992, LEST Foundation Technical Report No. 54, Univ. Oslo

Stenflo, J. O., Bianda, M., Keller, C. U., \& Solanki, S. K. 1997, A\&A, 322, 985

Stenflo, J. O., Keller, C. U., Gandorfer, A. 1998, A\&A, 329, 319

Stenflo, J. O., Gandorfer, A., \& Keller, C. U. 2000a, A\&A, 355, 781

Stenflo, J. O., Keller, C. U., \& Gandorfer, A. 2000b, A\&A, 355, 789

Stenflo, J. O., Gandorfer, A., Wenzler, T., \& Keller, C. U. 2001, A\&A, 367, 1033

Trujillo Bueno, J. 2001, in Advanced Solar Polarimetry: Theory, Observations and Instrumentation, ed. M. Sigwarth, ASP Conf. Ser., 236, 161

Trujillo Bueno, J., Collados, M., Paletou, F., \& Molodij, G. 2001, in Advanced Solar Polarimetry: Theory, Observations and Instrumentation, ed. M. Sigwarth, ASP Conf. Ser., 236, 141

Trujillo Bueno, J., Casini, R., Landolfi, M., \& Landi Degl'Innocenti, E. 2002, ApJL, 566, L53 